\title{
Fatores associados a quedas em idosos com catarata
}

\author{
Factors associated with falls in older adults with cataracts
}

Leonardo Petrus da Silva Paz ${ }^{1}$

Larissa de Lima Borges ${ }^{2}$

Vera Regina Fernandes da Silva Marães ${ }^{1}$

Marília Miranda Forte Gomes ${ }^{3}$

Maria Márcia Bachion ${ }^{4}$

Ruth Losada de Menezes ${ }^{5}$

${ }^{1}$ Faculdade de Ceilândia,

Universidade de Brasília

(UnB). Universidade de

Brasília,. QNN14 AE CEI-

Sul, Guariroba. 72220-

140 Brasília DF Brasil.

leopetruspaz@gmail.com

${ }^{2}$ Secretaria do Estado de

Saúde do Distrito Federal.

Brasília DF Brasil.

${ }^{3}$ Programa de Pós-

Graduação em Engenharia

Biomédica, UnB. Brasília

DF Brasil.

${ }^{4}$ Faculdade de Enfermagem,

Universidade Federal de

Goiás. Goiânia GO Brasil.

${ }^{5}$ Programa de Pós-

Graduação em Ciências e

Tecnologias em Saúde, UnB.

Brasília DF Brasil.
Abstract The aim of the present study was to evaluate factors associated with falls in community-dwelling older adults diagnosed with cataracts. An analytical, cross-sectional study was conducted with a sample of community-dwelling older adults residing in the Federal District of Brazil. Interviews and assessment tools were administered, such as the Timed Up and Go test, Short Physical Performance Battery (SPPB), Biodex Balance System, Katz Index, Lawton Scale, Minnesota Leisure Time Physical Activity Questionnaire and Mini Mental State Examination. Statistical analysis involved binary logistic regression. One hundred forty-two older adults (85 with cataracts) participated in the study (mean age: $69.39 \pm 5.67$ years). Falls were associated with the female sex (OR: 4.45) and sub-maximum score on the SPPB (OR: 3.53) among patients with cataracts, whereas multimorbidity (OR: 5.10) was the risk factor risk for older adults without cataracts. The data suggest different risk factors for falls among older adults diagnosed with cataracts.

Key words Accidental falls, Cataract, Aged, Postural balance, Physical fitness
Resumo O objetivo deste artigo é avaliar os fatores associados a quedas em idosos comunitários com diagnóstico de catarata. Trata-se de pesquisa transversal e analítica. A amostra se constituiu de idosos comunitários residentes no Distrito Federal, Brasil. Foram aplicados os seguintes instrumentos: questionário sociodemográfico; Timed Up and Go; Short Battery Performance Test; a Plataforma de Equilíbrio Biodex Balance System; o Índice de Katz, escala de Lawton; Mini-Exame do Estado Mental; e os critérios de fragilidade de John Hopkins Medical Institutions. Para análise empregou-se regressão logística binária. Participaram 142 idosos (85 destes com catarata), com média de idade de 69,39 anos ( \pm 5,67). Observou-se associação das quedas com sexo feminino $(O R: 4,45) e$ pontuação submáxima no SPPB (OR:3,53) entre os pacientes com catarata, ao passo que a presença de multimorbidades (OR:5,10) foi o fator de risco para os idosos sem catarata. Os dados sugerem fatores de risco diferentes para idosos com diagnóstico de catarata.

Palavras-chave Acidentes por quedas, Catarata, Idoso, Equilíbrio postural, Aptidão física 


\section{Introdução}

A catarata é a causa mais comum de cegueira no mundo e é uma das doenças degenerativas mais prevalentes em idosos ${ }^{1}$. Estima-se que no mundo, entre as 20 milhões de pessoas acometidas por deficiência visual severa, um terço dos casos seja resultado da catarata ${ }^{2}$, e segundo a Pesquisa Nacional de Saúde 2013, a ocorrência dessa doença no Brasil em idosos atingiu 28,7\% ${ }^{3}$.

A catarata pode agravar o equilíbrio postural, a mobilidade e a capacidade funcional, além de estar relacionada ao medo de quedas, ao maior número de morbidades, a alterações de humor, ao declínio cognitivo e ao pobre desempenho nos testes de equilíbrio postural e mobilidade ${ }^{4,5}$. Em concordância, o equilíbrio postural depende da correta integração das informações sensoriais vestibulares, proprioceptivas e visuais. A deficiência em um destes sistemas sensoriais pode ocasionar deficiência nos ajustes no equilíbrio postural $^{6,7}$.

Idosos com catarata apresentam um borramento da visão, ocasionando diminuição na acuidade visual, na sensibilidade ao contraste e na percepção de cor $^{1}$. Quando associada à pouca iluminação ambiental, a catarata favorece a ocorrência de escorregões e derrapadas em superfícies irregulares do chão do ambiente (ex.: poças, pontas de tapetes erguidas, batentes de portas) $)^{8}$. Estudos têm evidenciado a associação de uma ou mais quedas em idosos com deficiências visuais, uma vez que fatores como a pobre acuidade visual, a sensibilidade de contraste reduzida e a diminuição do campo visual estão associados a ocorrência de quedas ${ }^{8,9}$.

$\mathrm{Na}$ literatura há consenso quanto à significativa melhora na acuidade visual após a cirurgia de catarata ${ }^{10,11}$. Entretanto, o efeito da cirurgia sobre o risco de quedas é inconclusivo ${ }^{10}$, pois há relatos de redução ${ }^{12}$, mas também de aumento desse risco ${ }^{11}$. Outros fatores, tais como estilo de vida, o uso de medicação, as multimorbidades e os níveis de atividade física podem influenciar o risco de quedas em idosos com catarata, mesmo após a recuperação da visão ${ }^{11}$.

Além da deficiência visual, outros fatores intrínsecos e extrínsecos podem estar relacionados ao risco de quedas em idosos comunitários, tais como a incontinência urinária, a depressão, o medo de quedas, anormalidades do equilíbrio, deficiência cognitiva, polifarmácia, histórico de quedas, limitação funcional, entre outros ${ }^{13}$. De fato, são abundantes na literatura investigações que abordam os aspectos multidimensionais do risco de quedas em idosos comunitários ${ }^{14,15}$ e idosos de instituições de longa permanência ${ }^{16,17}$.

Contudo, são pouco estudados os grupos com problemas específicos, tais como idosos com catarata. São necessários, por isso, estudos adicionais, para investigar a relação entre a deficiência visual e as quedas, pois muitos estudos não levam em consideração a influência das múltiplas variáveis confundidoras que podem estar associadas às quedas ${ }^{18}$.

Esta investigação partiu da hipótese de que idosos com catarata apresenta fatores de risco diferentes de idosos sem esse diagnóstico. Neste contexto, o objetivo do presente trabalho foi avaliar os fatores associados a quedas em idosos comunitários com diagnóstico de catarata.

\section{Métodos}

Esta pesquisa de corte transversal, analítica e observacional foi realizada no Distrito Federal (DF), Brasil. Foram avaliados 142 idosos comunitários, recrutados por meio de busca ativa em programas de envelhecimento ativo e saudável da cidade de Ceilândia e em serviços de oftalmologia de dois hospitais públicos do Distrito Federal.

Foram incluídos idosos com 60 anos ou mais de ambos os sexos, com diagnóstico médico de catarata bilateral confirmado por meio de técnicas padronizadas ${ }^{1}$. Foram adotados os seguintes critérios de exclusão: diagnóstico clínico de doenças neurológicas ou de deficiência visual não corrigida e pontuação no Mini-Exame do Estado Mental sugestiva de deficiência cognitiva (ponto de corte ajustado pela escolaridade) ${ }^{19}$. Foram excluídos ainda os idosos que apresentaram ao exame paralisias ou alterações ortopédicas (tais como amputações e fraturas), ou incapacidade para se manter em ortostatismo e de se locomover sem assistência.

A pesquisa foi realizada no período de dezembro de 2011 a dezembro de 2012 e atendeu às recomendações para pesquisa envolvendo seres humanos, com aprovação do projeto pelo Comitê de Ética da Faculdade de Ensino e Pesquisa em Ciências da Saúde (FEPECS), Brasília, DF, Brasil. Esta pesquisa faz parte de um estudo maior denominado: "Impacto da cirurgia de catarata na ocorrência de quedas e nos aspectos multidimensionais de saúde: estudo longitudinal de idosos no Distrito Federal, Brasil”.

A variável desfecho "queda", definida como um evento não esperado em que o indivíduo cai até o chão ou a um nível inferior ${ }^{20}$, foi avaliada 
por meio de autorrelato dos participantes nos últimos 12 meses.

As demais variáveis e os respectivos métodos de avaliação incluíram:

- Dados sociodemográficos: abrangeram idade, sexo, estado civil, se lê e escreve e nível de escolaridade, informações sobre as condições de saúde (presença ou não de diagnóstico de catarata, realização de cirurgia de catarata, presença de comorbidades, depressão, número de medicações, uso de psicotrópicos) e estilo de vida (tabagismo, atividade física e alcoolismo). A variável multimorbidade foi definida como a presença de 5 ou mais doenças crônico-degenerativas (tais como diabetes, hipertensão arterial, doenças renais, cardiovasculares, depressão, osteoporose, entre outras). Estes dados foram obtidos por meio de aplicação de questionário estruturado;

- Nível de atividade física: foi determinado por meio da versão brasileira do Minnesota Leisure Time Activity Questionnaire ${ }^{21} \mathrm{e}$ da classificação do idoso como sedentário ou ativo, foi baseada no critério do American College of Sports Medicine (ACSM). Considera-se ativo o idoso que realiza pelo menos 150 minutos semanais de atividade física de moderada intensidade (3 METs) ou então que demanda 75 minutos em atividades de intensidade vigorosa ou esportiva $(6 \mathrm{METs})^{22,23}$;

- Desempenho físico-funcional: esta avaliação foi realizada no Laboratório de Análise de Movimento da instituição utilizando-se a versão brasileira do Short Physical Performance Test $(\mathrm{SPPB})^{24}$ - para avaliar a força de membros inferiores, o equilíbrio corporal, a velocidade de marcha e o risco de queda; e o Timed Up and Go (Levante e ande cronometrado - TUG ${ }^{25}$, destinado à avaliação do equilíbrio e da capacidade funcional para marcha e risco de quedas. Foram determinados pontos de corte por análise Roc (análise não apresentada neste estudo) para risco de quedas nas variáveis SPPB (ordinal) e TUG (numérica), para constituírem variáveis binárias. O risco de quedas também foi avaliado utilizando-se a Plataforma de Equilíbrio Biodex Balance System (BBS Fall risk - Fri) ${ }^{26}$;

- Independência funcional: concerne ao desempenho de atividades de vida diária (AVDs), avaliado pela versão brasileira do Index of Activity Daily Living e de atividades instrumentais de vida diária (AIVDs) ${ }^{27}$, avaliado pela Escala de Lawton $^{28}$ validada para a língua portuguesa.

Para avaliar a associação da queda com as variáveis de interesse nos idosos com e sem catarata, foi conduzida a análise de regressão lo- gística binária para determinar a Odds Ratio (OR) utilizando-se o programa SPSS, versão 20. Inicialmente foram introduzidas, uma a uma, as variáveis candidatas a fator de risco e/ou proteção à ocorrência de queda (análise de regressão univariada, com método "inserir").

As variáveis que atingiram $\mathrm{p}<0,20$ foram incluídas em blocos de variáveis e agrupadas em características sociodemográficas, condições de saúde, desempenho físico-funcional e estilo de vida, para se obter um modelo mais representativo.

Finalmente, foi realizada análise multivariada a partir da divisão da amostra em grupos de pacientes com e sem diagnóstico de catarata, que constituíram-se os modelos A e B para cada um destes substratos da amostra. Todos os modelos foram ajustados para idade. Foi observada a regra de pelo menos dez eventos (sucessos ou fracassos) para cada variável independente do modelo. As diferenças entre os grupos foram avaliadas por meio dos testes estatísticos de Mann-Whitney e Qui Quadrado (para variáveis categóricas).

\section{Resultados}

Foram convidados para a avaliação 176 idosos e, destes, 144 compareceram; sendo excluídos dois pacientes: um por não enxergar o alvo na tela da plataforma de equilíbrio (BBS); o outro não soube relatar se caiu. A descrição das características da amostra $(n=142)$ consta na Tabela 1, considerando em separado o critério de diagnóstico de catarata. Não foram observadas diferenças estatisticamente significativas para as variáveis investigadas entre os extratos de pacientes com e sem catarata, exceto quanto às variáveis "acuidade visual" e risco de quedas no Teste Timed Up and Go com ponto de corte de 7.67 segundos $\left(\mathrm{TUG}_{7,67}\right)$.

Pode-se observar prevalência de quedas de $40 \%$, alta prevalência de prática de atividade física no nível recomendado (considerando todos os idosos) e ainda pontuações submáximas na avaliação do desempenho físico-funcional e da independência para suas AVDs e AIVDs. Houve uma tendência a uma maior prevalência de quedas no grupo de pacientes sem diagnóstico de catarata. Mas não foi atingida a significância estatística.

Na Tabela 2 consta o resultado da análise univariada, considerando os grupos com e sem diagnóstico de catarata e o resultado da análise das diferenças entre os grupos nas diferentes variáveis. As variáveis que individualmente se associaram às quedas foram destacadas com um 
Tabela 1. Características da amostra estudada, composta por idosos comunitários. Distrito Federal, Brasil, 2015.

\begin{tabular}{|c|c|c|c|c|c|c|c|}
\hline \multirow{2}{*}{ Variável } & \multicolumn{3}{|c|}{ Catarata $(n=85)$} & \multicolumn{4}{|c|}{ Sem catarata $(n=57)$} \\
\hline & $\mathbf{n}$ & $\%$ & Média $( \pm \mathrm{DP})$ & $\mathbf{n}$ & $\%$ & Média $( \pm \mathrm{DP})$ & $\mathbf{p}$ \\
\hline \multicolumn{8}{|l|}{ Quedas } \\
\hline Não & 52 & $61,18 \%$ & & 24 & $42,11 \%$ & & 0,70 \\
\hline $\operatorname{Sim}$ & 33 & $38,82 \%$ & & 33 & $57,80 \%$ & & \\
\hline \multicolumn{8}{|l|}{ Idade } \\
\hline$<75$ anos & 67 & $78,82 \%$ & & 51 & $89,47 \%$ & & 0,97 \\
\hline$>75$ anos & 18 & $21,18 \%$ & & 6 & $10,53 \%$ & & \\
\hline \multicolumn{8}{|l|}{ Sexo } \\
\hline Masculino & 31 & $36,47 \%$ & & 20 & $35,09 \%$ & & 0,86 \\
\hline Feminino & 54 & $63,53 \%$ & & 37 & $64,91 \%$ & & \\
\hline \multicolumn{8}{|l|}{ Estado civil } \\
\hline Casado ou vive com companheiro & 47 & $55,29 \%$ & & 29 & $50,88 \%$ & & 0,93 \\
\hline Solteiro & 8 & $9,41 \%$ & & 6 & $10,53 \%$ & & \\
\hline Divorciado ou separado & 8 & $9,41 \%$ & & 7 & $12,28 \%$ & & \\
\hline Viúvo & 22 & $25,88 \%$ & & 15 & $26,32 \%$ & & \\
\hline \multicolumn{8}{|l|}{ Ausência de cônjuge } \\
\hline Não & 47 & $55,29 \%$ & & 29 & $50,88 \%$ & & 0,60 \\
\hline $\operatorname{Sim}$ & 38 & $44,71 \%$ & & 28 & $49,12 \%$ & & \\
\hline \multicolumn{8}{|l|}{ Sabe ler e escrever } \\
\hline Não & 23 & $27,06 \%$ & & 10 & $17,54 \%$ & & \\
\hline Sim & 62 & $72,94 \%$ & & 47 & $82,46 \%$ & & 0,19 \\
\hline \multicolumn{8}{|l|}{ Multimorbidade } \\
\hline Não & 66 & $77,65 \%$ & & 43 & $75,44 \%$ & & \\
\hline Sim & 19 & $22,35 \%$ & & 14 & $24,56 \%$ & & 0,378 \\
\hline \multicolumn{8}{|l|}{ Uso de psicotrópico } \\
\hline Não & 66 & $88,00 \%$ & & 38 & $88,37 \%$ & & 0,95 \\
\hline Sim & 9 & $12,00 \%$ & & 5 & $11,63 \%$ & & \\
\hline \multicolumn{8}{|l|}{ Polifarmácia ( 3 ou mais) } \\
\hline Não & 31 & $36 \%$ & & 40 & $47 \%$ & & 0,28 \\
\hline Sim & 24 & $28 \%$ & & 45 & $53 \%$ & & \\
\hline \multicolumn{8}{|l|}{ Acuidade visual deficiente } \\
\hline Não & 53 & $62,40 \%$ & & 50 & $87,70 \%$ & & 0,01 \\
\hline Sim & 32 & $37,60 \%$ & & 7 & $12,30 \%$ & & \\
\hline
\end{tabular}

$(\mathrm{p}<0,05)$ ou dois asteriscos $(\mathrm{p}<0.20)$ em cada grupo. Foram utilizados os pontos de corte de 6,67 segundos no TUG $\left(\mathrm{TUG}_{7,67 \mathrm{~s}}\right.$ ) e de 11 pontos na escala SPPB $\left(\mathrm{SPPB}_{11}\right)$, Os modelos de regressão multivariada "A" $\mathrm{e}$ "B" representam o risco de quedas para os grupos, sem e com catarata, respectivamente, os quais foram ajustados para idade (idade superior a 75 anos) e sexo. Idosos do sexo feminino apresentaram quatro vezes mais chances de cair em relação aos do masculino. Adicionalmente, idosos com catarata que possuíam uma pontuação inferior a 11 pontos no teste de desempenho físico SPPB apresentaram 3,5 vezes mais chances de cair do que os que obtiveram pontuação superior. A multimorbidade foi o fator relacionado a quedas em pacientes sem diagnóstico de catarata (Tabela 3).

\section{Discussão}

Neste estudo foram investigados os fatores associados às quedas em idosos comunitários com diagnóstico de catarata, comparando-se aos fatores de risco para quedas em idosos sem este diagnóstico. Em síntese, foi observada uma maior 
Tabela 1. Características da amostra estudada, composta por idosos comunitários. Distrito Federal, Brasil, 2015.

\begin{tabular}{|c|c|c|c|c|c|c|c|}
\hline \multirow{2}{*}{ Variável } & \multicolumn{3}{|c|}{ Catarata $(n=85)$} & \multicolumn{4}{|c|}{ Sem catarata $(n=57)$} \\
\hline & $\mathbf{n}$ & $\%$ & Média $( \pm \mathrm{DP})$ & $\mathbf{n}$ & $\%$ & Média $( \pm \mathrm{DP})$ & $\mathbf{p}$ \\
\hline Índice de Katz & & & $0,47( \pm 0,52)$ & & & $0,32( \pm 0,46)$ & 0,19 \\
\hline Escala de Lawton & & & $24,20( \pm 3,29$ & & & $25,26( \pm 2,83)$ & 0,60 \\
\hline Mini-Exame do Estado Mental & & & $24,33( \pm 3,36)$ & & & $24,84( \pm 3,28)$ & 0,5 \\
\hline \multicolumn{8}{|l|}{ Número de critérios de fragilidade } \\
\hline Não frágil & 32 & $37,65 \%$ & & 14 & $24,56 \%$ & & 0.26 \\
\hline Pré-frágil & 52 & $61,18 \%$ & & 42 & $73,68 \%$ & & \\
\hline Frágil & 1 & $1,18 \%$ & & 1 & $1,75 \%$ & & \\
\hline \multicolumn{8}{|l|}{$\begin{array}{l}\text { Nível de atividade física } \\
\text { recomendado }\end{array}$} \\
\hline Ativo & 45 & $52,90 \%$ & & 31 & $54,40 \%$ & & 0,20 \\
\hline Não ativo & 40 & $47,10 \%$ & & 26 & $45,60 \%$ & & \\
\hline \multicolumn{8}{|l|}{ Risco de quedas (BBS Fri) } \\
\hline Baixo risco & 62 & $72,94 \%$ & & 44 & $77,20 \%$ & & 0,57 \\
\hline Elevado risco & 23 & $27,06 \%$ & & 13 & $22,80 \%$ & & \\
\hline \multicolumn{8}{|l|}{ TUG - ponto de corte 7,67 segundos } \\
\hline Baixo risco ( $<7,67$ segundos $)$ & 44 & $51,80 \%$ & & 39 & $68,40 \%$ & & 0,04 \\
\hline Elevado risco & 41 & $48,20 \%$ & & 18 & $31,60 \%$ & & \\
\hline \multicolumn{8}{|l|}{ SPPB - ponto de corte de 11 pontos } \\
\hline Baixo risco ( $<11$ pontos $)$ & 39 & $45,90 \%$ & & 35 & $61,40 \%$ & & 0,07 \\
\hline Elevado risco & 46 & $54,10 \%$ & & 22 & $38,60 \%$ & & \\
\hline
\end{tabular}

chance de quedas entre os pacientes com catarata do sexo feminino e com uma pontuação de 11 pontos no SPPB. Enquanto que entre os idosos sem catarata ter 5 ou mais doenças foi o fator associado a uma maior chance de quedas.

No Brasil são escassos os estudos analíticos que envolvem idosos com catarata. Estudos prévios haviam apresentado contribuições quanto à relação da catarata com o equilíbrio postural, o medo de quedas e a qualidade de vida ${ }^{29,30}$, mas nestes estudos não foram observadas outras dimensões do fenômeno "quedas” nessa população, limitando-se à descrição de características sociodemográficas ${ }^{31,32}$. No contexto da literatura internacional são escassos estudos multidimensionais abordando a relação entre a deficiência visual e as quedas ${ }^{18}$.

A deficiência visual poderia ocasionar dificuldades para detectar obstáculos com pouco contraste, ou para julgar distâncias, ou para perceber relações espaciais, e ainda para processar toda a informação visual necessária aos ajustes no controle postural e para mobilidade, dificultando a adaptação às demandas ambientais necessárias à execução das atividades cotidianas ${ }^{9,7}$. O impacto da deficiência visual sobre as quedas pode ser maior se acompanhado por outras deficiências sensoriais e no equilíbrio ${ }^{33}$.

Um desempenho prejudicado em testes visuais está relacionado a pior desempenho em testes físico-funcionais ${ }^{34}$. Idosos com catarata e com relato de medo de cair apresentam menor confiança no seu equilíbrio para evitar quedas e podem limitar suas atividades funcionais por medo de cair ${ }^{30}$. Há uma lacuna de conhecimento envolvendo investigação do efeito das deficiências visuais no nível de atividade física dos idosos. Sabe-se que a cirurgia de catarata é eficaz na melhora da acuidade visual ${ }^{10}$, mas é incerto se há redução no risco de quedas ${ }^{10-12}$.

Neste contexto, a catarata é considerada um fator de risco independente para quedas ${ }^{9}$ e pode determinar prejuízos mais acentuados no equilíbrio e na mobilidade, em relação aos demais ido- 
Tabela 2. Regressão logística univariada de variáveis candidatas a fator de risco ou proteção para quedas. Distrito Federal, Brasil.

\begin{tabular}{|c|c|c|c|c|c|}
\hline \multirow{2}{*}{ Variável } & \multirow{2}{*}{ Significado } & \multicolumn{2}{|c|}{ Catarata $(n=85)$} & \multicolumn{2}{|c|}{ Sem Catarata $(n=57)$} \\
\hline & & p valor & OR & p valor & OR \\
\hline \multirow[t]{2}{*}{ Idade superior a 75 anos } & Não & & & & \\
\hline & Sim & 0,58 & 1,34 & 0,68 & 1,40 \\
\hline \multirow[t]{2}{*}{ Sexo } & Masculino & & & & \\
\hline & Feminino & $0,02^{*}$ & 5,60 & 0,74 & 0,83 \\
\hline \multirow[t]{2}{*}{ Ausência de cônjuge } & Não & & & & \\
\hline & Sim & $0,02^{\star}$ & 2,90 & 0,51 & 1,42 \\
\hline \multirow[t]{2}{*}{ Diagnóstico de catarata } & Não & & & & \\
\hline & Sim & & & & \\
\hline \multirow[t]{2}{*}{ Utiliza psicotrópico } & Não & & & & \\
\hline & Sim & $0,08^{\star *}$ & 3,73 & $0,17^{\star \star}$ & 4,94 \\
\hline \multirow[t]{2}{*}{ Polifarmácia ( 3 ou mais) } & Não & & & & \\
\hline & Sim & $0,05^{\star}$ & 2,52 & 0,79 & 0,87 \\
\hline \multirow[t]{2}{*}{ Multimorbidade } & Não & & & & \\
\hline & $\operatorname{Sim}$ & 0,39 & 1,58 & $0,06^{\star *}$ & 3,36 \\
\hline Índice de Katz (AVDs) & Independência em AVDs & $0,02^{\star}$ & 4,14 & 0,74 & 0,82 \\
\hline Escala de Lawton (AIVDs) & Independência em AIVDs & $0,06^{\star *}$ & 0,87 & 0,32 & 1,15 \\
\hline \multirow[t]{2}{*}{ Risco de quedas (BBS Fri) } & Baixo risco de quedas & 0,34 & 0,60 & 0,73 & 1,24 \\
\hline & Elevado risco de quedas & $0,04^{\star}$ & 3,73 & 0,23 & 0,72 \\
\hline $\begin{array}{l}\text { Teste Levante e Ande } \\
\text { Cronometrado (TUG) }\end{array}$ & Tempo em segundos no TUG & 0,40 & 1,07 & 0,53 & 1,14 \\
\hline \multirow{2}{*}{$\begin{array}{l}\text { Risco de quedas (ponto de } \\
\text { corte de } 7,67 \text { segundos - } \\
\text { TUG) }\end{array}$} & Baixo risco de quedas & & & & \\
\hline & Elevado risco de quedas & $0,17^{\star *}$ & 1,85 & 0,41 & 1,60 \\
\hline \multirow{2}{*}{$\begin{array}{l}\text { SPPB (ponto de corte de } 11 \\
\text { pontos) }\end{array}$} & Baixo risco de quedas & & & & \\
\hline & Elevado risco de quedas & $0,02^{\star}$ & 4,16 & 0,88 & 0,92 \\
\hline \multirow{2}{*}{$\begin{array}{l}\text { Nível de atividade física } \\
\text { recomendado }\end{array}$} & Idoso ativo & & & & \\
\hline & Idoso não ativo & $0,09^{\star *}$ & 2,11 & $0,05^{\star}$ & 3,12 \\
\hline
\end{tabular}

$\operatorname{sos}^{5}$, o que poderia justificar um pior desempenho nos testes de equilíbrio, e por consequência uma maior prevalência de quedas neste extrato da amostra.

Esse fato não foi observado nos resultados apresentados, pois não houve diferenças estatisticamente significativas na prevalência de quedas entre os grupos. Houve uma tendência a uma maior prevalência de quedas no grupo sem catarata, embora idosos com catarata tenham apresentado pior desempenho nos testes físico-funcionais. A prevalência de quedas entre os idosos sem catarata pode estar associada à presença de multimorbidades, uma vez que a presença de cinco ou mais comorbidades neste extrato aumentou em cinco vezes a chance de cair. As multimorbidades podem estar associadas a quedas ${ }^{35} \mathrm{e}$ a condição de fragilidade, que por sua vez podem aumentar ao longo do tempo o risco a desfechos desfavoráveis tais como quedas, hospitalização e fraturas ${ }^{36}$.

Apesar desta aparente diferença de prevalência de quedas entre os grupos estudados, não foram encontradas diferenças entre os pacientes com diagnóstico de catarata e os sem em todas as variáveis aqui investigadas, exceto nas variáveis acuidade visual e no teste Timed Up and Go.

Em concordância, em estudo prévio do tipo caso-controle realizado na Malásia envolvendo idosos que haviam sofrido fraturas de quadril, não foi reportada diferença entre os casos de catarata e os normais (controle) quanto ao estado pré-mórbido, ao grau de independência para mobilidade e uso de dispositivos auxiliares 
Tabela 3. Modelos de regressão representando as quedas e fatores associados de idosos com e sem diagnóstico de catarata, Distrito Federal, Brasil.

\begin{tabular}{|c|c|c|c|c|c|c|c|}
\hline Modelo & Possui catarata & Variável & B & Erro padrão & Wald & $\mathbf{P}$ & OR \\
\hline \multirow[t]{5}{*}{$\mathrm{A}$} & Não & Idade de 75 ou mais & 0.76 & 1.06 & 0.52 & 0.47 & 2.15 \\
\hline & & Sexo & -0.35 & 0.79 & 0.2 & 0.66 & 0.71 \\
\hline & & Multimorbidade (5 e mais) & 1.63 & 0.81 & 4.08 & $0,04^{\star}$ & 5.1 \\
\hline & & Psicotrópico & 1.61 & 1.23 & 1.71 & 0.19 & 5.01 \\
\hline & & Constante & -0.46 & 0.74 & 0.39 & 0.54 & 0.63 \\
\hline \multirow[t]{7}{*}{ B } & Sim & Idade superior a 75 anos & 0.24 & 0.7 & 0.12 & 0.73 & 1.28 \\
\hline & & Sexo & 1.49 & 0.65 & 5.22 & $0,02^{*}$ & 4.45 \\
\hline & & Ausência de cônjuge & 0.62 & 0.53 & 1.36 & 0.24 & 1.85 \\
\hline & & Polifarmácia ( 3 ou mais) & 0.85 & 0.54 & 2.47 & 0.12 & 2.34 \\
\hline & & Desempenho físico (SPPB11) & 1.26 & 0.6 & 4.48 & $0,03^{*}$ & 3.53 \\
\hline & & Nível de atividade física recomendado & -0.08 & 0.58 & 0.02 & 0.89 & 0.92 \\
\hline & & Constante & -3.04 & 0.73 & 17.13 & 0.01 & 0.05 \\
\hline
\end{tabular}

Legenda: $\mathrm{OR}^{\star}=$ Odds Ratio ajustada; MEEM = Mine Exame do Estado Mental; AVD’s = Atividades de vida diária; MET =

coeficiente metabólico. $\mathrm{O}\left({ }^{*}\right)$ representa o valor de $\mathrm{p} \leq 0,05$; enquanto que ${ }^{* *}$ ) representa o valor de $\mathrm{p} \leq 0,20$.

nem quanto à variável morar sozinho ${ }^{37}$. Por outro lado, idosos escoceses com deficiência visual atendidos na atenção primária à saúde apresentaram mais comorbidades em relação a idosos sem esse tipo de deficiência ${ }^{38}$. A diferença entre os estudos pode estar relacionada ao local de recrutamento: no primeiro a amostra foi recrutada em ambiente hospitalar e no segundo na atenção primária à saúde.

Um achado interessante da presente pesquisa foi a observação de uma elevada prevalência de quedas em idosos comunitários com altos níveis de funcionalidade, constatada pelos altos níveis de atividade física e pelas pontuações submáximas de desempenho físico-funcional e de independência para suas AVDs e AIVDs.

Considera-se que um desempenho ruim em testes de equilíbrio como o TUG é associado ao histórico de quedas ${ }^{39}$, e este teste tem sido utilizados para triagem de idosos com risco de quedas, embora não haja consenso sobre o ponto de corte ideal para identificar idosos com risco de quedas ${ }^{40}$. Recentemente o uso do TUG tem sido questionado quanto à sua capacidade preditiva para quedas em idosos ${ }^{41}$. Sabe-se que idosos praticantes de mais altos níveis de atividade física obtêm melhores pontuações nos testes de equilíbrio postural ${ }^{42}$, e podem apresentar "efeito teto nas pontuações nos testes de equilíbrio ${ }^{40,43}$.

Em concordância os participantes aqui avaliados obtiveram pontuações elevadas nos testes de desempenho físico-funcional (TUG, SPPB e BBS). Ressalta-se que a adequação dos pontos de corte dos testes de equilíbrio ao perfil da população avaliada é fundamental. No presente estudo mesmo realizando adequações no pontes de corte dos testes de desempenho físico funcional para melhorar a sensibilidade e especificidade ${ }^{42}$, os instrumentos de avaliação do equilíbrio postural como TUG e BBS não foram associados às quedas nos grupos aqui investigados. Acrescenta-se que os testes físico funcionais utilizados no presente estudo não incluem a avaliação dos componentes sensoriais do equilíbrio postural ${ }^{43}$.

Em conjunto, estes achados da literatura sugerem que uma pontuação elevada nos testes físicos funcionais não implica necessariamente uma ausência do risco de cair. Sujeitos com altos níveis de funcionalidade e com ausência de deficiências no equilíbrio postural continuam a realizar suas atividades habituais e assim poderiam se expor a situações de risco para quedas ${ }^{44}$.

Deste modo, a alta prevalência de atividade física entre os idosos da amostra poderia explicar a alta prevalência de quedas na amostra estudada no presente estudo. Entre os participantes, 41\% relataram praticar atividade física em níveis recomendados $^{19}$. Mesmo entre os idosos com diagnóstico de catarata, este percentual foi de 52\%. Esses são superiores aos achados da Pesquisa Nacional de Saúde, 2013, na qual a proporção de idosos praticantes de atividade física no nível recomendado no Brasil totalizou 13,6\%

A prática de atividade física pode reduzir o risco de quedas ${ }^{46}$, porém ao praticá-la em ambiente externo, os idosos podem ser expostos a 
barreiras ambientais ${ }^{47}$. Mas há controvérsias. Por exemplo, Okubo et al. investigaram se o hábito de praticar a caminhada aumentaria o risco de quedas $^{48}$. Esses autores sugerem que a prática de caminhada não está relacionada a quedas em idosos com baixo risco de quedas, mas, entre os que possuem dois ou mais fatores de risco para tal, as caminhadas estão fortemente associadas a estes desfechos.

Foi possível notar diferentes fatores de risco para quedas nos modelos de regressão para idosos com e sem catarata. No grupo com catarata, o sexo e o desempenho físico permaneceram no modelo final como fatores associados a quedas.

As mulheres com catarata apresentaram cinco vezes mais chance de cair do que os homens com catarata. Isso talvez ocorra porque as mulheres sofrem declínio sensorial, motor e cognitivo mais precocemente do que os homens ${ }^{49}$, estando mais próximas do limite no qual a potência muscular poderia comprometer o estado funciona $\mathrm{l}^{50}$; ou ainda pelo maior uso de serviços de saúde por partes das idosas, incluindo consultas médicas, o que viabiliza a constatação de maior número de diagnósticos de catarata ${ }^{51}$; ou pela maior longevidade e consequente acúmulo de doenças relacionadas ao envelhecimento; ou ainda porque as mulheres procuram mais a cirurgia de catarata ${ }^{52}$. Diferenças similares entre homens e mulheres quanto ao impacto funcional da catarata já havia sido reportadas ${ }^{53}$. Em geral considera-se que as mulheres sentem mais medo de cair do que os homens, e este medo supostamente poderia exercer um papel protetor para as quedas ${ }^{54}$. A disparidade entre homens e mulheres pode estar ligada ao fator cultural, pois as mulheres (mesmo àquelas que sofreram quedas) continuam exercendo seu papel social, realizando atividades em seu ambiente domiciliar ao longo do tempo ${ }^{52}$.

No substrato constituído exclusivamente por pacientes com catarata, a pontuação inferior a 11 pontos no SPPB foi considerada um fator de risco para quedas. O equilíbrio é mais prejudicado quanto maior a deficiência visual; e, estes fatores juntos, portanto, tendem a resultar em maior incidência de quedas ${ }^{4,55}$. Nesses dois estudos foi utilizada a escala de equilíbrio de Berg.

O teste SPPB foi a única medida de desempenho físico-funcional associada às quedas entre os pacientes com catarata. Talvez porque o SPPB seja um teste mais abrangente por contemplar testes de força muscular, equilíbrio corporal e velocidade de marcha. Sugere-se que o SPPB, entre os testes utilizados, seja o mais adequado para avaliar idosos com diferentes níveis de funcionalidade, uma vez que pode diferenciar idosos mais ativos dos sedentários ${ }^{56}$, assim como detectar diferenças de desempenho entre frágeis e não frágeis ${ }^{57}$. Em estudo de base populacional foi observada diferença estatisticamente significativa no SPPB entre caidores recorrentes e não recorrentes ${ }^{58}$.

Considerando os achados do presente estudo e a inexistência de evidências a favor da cirurgia de correção para catarata como estratégia única para redução do risco de quedas ${ }^{59,60}$, aumenta-se a importância do acompanhamento multidisciplinar dos idosos com diagnóstico de catarata para os orientar quanto às intervenções nos fatores de risco modificáveis, pois as estratégias multidimensionais são eficazes na redução do risco e na taxa de quedas ${ }^{59}$.

A avaliação multidimensional das quedas é fundamental. Certas patologias podem levar a quedas por interferirem no controle postural, como as que trazem prejuízo à visão (por exemplo, a catarata), ou por acarretarem deficiência na sensibilidade, como ocorre no diabetes mellitus ${ }^{61}$.

No contexto da avaliação multiprofissional, é importante notar ainda que as variáveis relacionadas ao uso de medicações foram os fatores associados às quedas observados em ambos os extratos da amostra sem, no entanto, atingir a significância estatística.

As limitações da presente pesquisa estão relacionadas ao corte transversal e à impossibilidade de adotar uma amostragem probabilística. Foram realizadas diversas análises estratificadas a fim de verificar a existência de fatores de confusão. Acrescenta-se que a escolha dos modelos de regressão foi realizada com base em estatísticas de aderência dos modelos aos dados.

Os resultados do presente estudo sugerem que idosos com altos níveis de funcionalidade podem obter pontuações submáximas em testes de equilíbrio e ainda assim apresentar uma alta prevalência de quedas.

Estes achados podem contribuir para o desenvolvimento de novas ideias a respeito da avaliação de idosos comunitários ativos, incluindo o uso de instrumentos multidimensionais para triagem no contexto da atenção primária à saú$\mathrm{de}^{62}$. Medidas simples na atenção primária como distribuição de óculos e triagens na comunidade com testes de acuidade visual podem ajudar no manejo de pacientes com catarata ${ }^{63}$.

Faz-se necessário, também, propiciar uma formação dos profissionais da saúde que contemple a promoção da saúde funcional do idoso em sua integralidade, rompendo com a fragmentação do trabalho dos profissionais da área ${ }^{64}$. 


\section{Conclusão}

Os dados sugerem que o sexo e o desempenho físico interferem no risco de quedas de idosos comunitários com diagnóstico de catarata. Entre os idosos sem diagnóstico de catarata a multimorbidade foi fator associado a quedas.

Os testes de desempenho físico, incluindo os testes relacionados ao equilíbrio postural, não devem ser utilizados isoladamente para identificação de idosos caidores, ainda que sejam feitos ajustes nas pontuações.

Estudos posteriores longitudinais são necessários para confirmar estes achados e assim contribuir para o desenvolvimento de estratégias multidisciplinares para avaliação do risco de quedas em idosos comunitários com diagnóstico de catarata.

\section{Colaboradores}

Os autores LL Borges, LPS Paz e RL Menezes trabalharam na pesquisa de campo, sendo que esta última autora atuou como a orientadora da pesquisa. LPS Paz, LL Borges, VRFS Marães, MMF Gomes, MM Bachion e RL Menezes participaram na redação final do manuscrito.

\section{Agradecimentos}

Ao CNPq pelo financiamento. 


\section{Referências}

1. Thompson J, Lakhani N. Cataracts. Prim Care Clin Off Pract 2015; 42(3):409-423.

2. Brian G, Taylor H. Cataract blindness--challenges for the 21st century. Bull World Health Organ 2001; 79(3):249-256.

3. Instituto Brasileiro de Geografia e Estatística (IBGE), Barbosa N, Menezes AP, Carlos A, Nardi F. Pesquisa Nacional De Saude 2013 - Ciclos De Vida. Rio de Janeiro: IBGE; 2013.

4. Aartolahti E, Häkkinen A, Lönnroos E, Kautiainen H, Sulkava R, Hartikainen S. Relationship between functional vision and balance and mobility performance in community-dwelling older adults. Aging Clin Exp Res 2013; 25(5):545-552.

5. Borges LDL, Santos FPV, Pagotto V, Menezes RL. Functional disability in community-dwelling elderly: the role of cataracts and contextual factors. Fisioter em Mov 2014; 27(2):189-200.

6. Pasma JH, Engelhart D, Maier AB, Schouten AC, van der Kooij H, Meskers CGM. Changes in sensory reweighting of proprioceptive information during standing balance with age and disease. J Neurophysiol 2015; 114(6):3220-3233.

7. Horak FB. Postural orientation and equilibrium: What do we need to know about neural control of balance to prevent falls? Age Ageing 2006; 35(Supl. 2):7-11.

8. Reed-Jones RJ, Solis GR, Lawson K, Loya AM, Cude-Islas D, Berger CS. Vision and falls: A multidisciplinary review of the contributions of visual impairment to falls among older adults. Maturitas 2013; 75(1):22-28.

9. Lord SR, Smith ST, Menant JC. Vision and falls in older people: Risk factors and intervention strategies. Clin Geriatr Med 2010; 26(4):569-581.

10. Desapriya E, Subzwari S, Scime-Beltrano G, Samayawardhena L, Pike I. Vision improvement and reduction in falls after expedited cataract surgery. Systematic review and metaanalysis. J Cataract Refract Surg 2010; 36(1):13-19.

11. Meuleners LB, Lee AH, Ng JQ, Morlet N, Fraser ML. First Eye Cataract Surgery and Hospitalization from Injuries Due to a Fall: A Population-Based Study. J Am Geriatr Soc 2012; 60(9):1730-1733.

12. Gillespie LD, Robertson MC, Gillespie WJ, Sherrington C, Gates S, Clemson LM, Lamb SE. Interventions for preventing falls in older people living in the community. Cochrane Database Syst Rev 2012; (9):CD007146.

13. Frith J, Davison J. Falls. Rev Clin Gerontol 2013; 23(02):101-117.

14. Rodrigues IG, Fraga GP, Barros MBDA. Falls among the elderly: risk factors in a population-based study. Rev Bras Epidemiol 2014; 17(3):705-718.

15. Deandrea S, Bravi F, Turati F, Lucenteforte E, La Vecchia C, Negri E. Risk factors for falls in older people in nursing homes and hospitals. A systematic review and meta-analysis. Arch Gerontol Geriatr 2013; 56(3):407-415.

16. Menezes RL, Bachion MM. Estudo da presença de fatores de riscos intrínsecos para quedas, em idosos institucionalizados. Cien Saude Colet 2008; 13(4):12091218.

17. Menezes RL, Bachion MM, Souza JT, Nakatani AYK. Estudo longitudinal dos aspectos multidimensionais da saúde de idosos institucionalizados. Rev Bras Geriatr Gerontol 2011; 14(3):485-496.
18. Salonen L, Kivel SL. Eye diseases and impaired vision as possible risk factors for recurrent falls in the aged: A systematic review. Curr Gerontol Geriatr Res 2012; 2012:1-10.

19. Brucki SMD, Nitrin R, Caramelli P, Bertolucci PHF, Okamoto IH. Sugestões para o uso do mini-exame do estado mental no Brasil. Arq Neuropsiquiatr 2003; 61(3 B):777-781.

20. Lamb SE, Jørstad-Stein EC, Hauer K, Becker C. Development of a common outcome data set for fall injury prevention trials: The Prevention of Falls Network Europe consensus. J Am Geriatr Soc 2005; 53(9):16181622.

21. Lustosa LP, Pereira DS, Dias RC, Britto R, Parentoni A, Pereira L. Tradução e adaptação transcultural do Minnesota Leisure Time Activities Questionnaire em idosos. Geriatr Gerontol 2011; 5(2):57-65.

22. Costa TB, Neri AL. Medidas de atividade física e fragilidade em idosos: dados do FIBRA Campinas, São Paulo, Brasil. Cad Saude Publica 2011; 27(8):1537-1550.

23. Nelson ME, Rejeski WJ, Blair SN, Duncan PW, Judge JO, King AC, Macera CA, Castaneda-Sceppa C; American College of Sports Medicine; American Heart Association. Physical activity and public health in older adults: Recommendation from the American College of Sports Medicine and the American Heart Association. Circulation 2007; 116(9):1094-1105.

24. Nakano MM. Versão brasileira da short physical performance battery - sppb: adaptação cultural e estudo da confiabilidade [dissertação]. Campinas: Unicamp; 2007.

25. Podsiladlo SR. Timed Get Up and Go Test. J Am Geriactrics Soc 1991; 29(2):2006.

26. Parraca J, Olivares PR, Carbonell-Baeza A, Aparicio V, Adsuar JC, Gusi N. Test-retest reliability of biodex balance SD on physically active old people. J Hum Sport Exerc 2011; 6(2):444-451.

27. Lino VTS, Pereira SRM, Camacho LAB, Ribeiro Filho ST, Buksman S. Cross-cultural adaptation of the Independence in Activities of Daily Living Index (Katz Index). Cad Saude Publica 2008; 24(1):103-112.

28. Santos RL, Virtuoso Júnior JS. Confiabilidade da versão brasileira da Escala de Atividades Instrumentais da Vida Diária. Rev Bras Promoção da Saúde 2008; 21(1):290-296.

29. Macedo BG, Pereira LSM, Rocha FL, Castro ANBV. Association between funcional vision, balance and fear of falling in older adults with cataracts. Rev Bras Geriatr e Gerontol 2012; 15(2):265-274.

30. Macedo BG, Pereira LSM, Rocha FL, Castro ANBV. Medo de cair e qualidade de vida em idosos com catarata. Rev Bras Geriatr e Gerontol 2013; 16(3):569577.

31. Carlos GA, Schellini SA, Espindola RF, Lana FP, Rodrigues AC, Padovani CR. Cataract prevalence in Central-West region of Sao Paulo State, Brazil. Arq Bras Oftalmol 2009; 72(3):375-379.

32. Silva LMP, Muccioli C, Belfort Júnior R. Perfil socioeconômico e satisfação dos pacientes atendidos no mutirão de catarata do Instituto da Visão - UNIFESP. Arq Bras Oftalmol 2004; 67(5):737-744. 
33. Kulmala J, Viljanen A, Sipilä S, Pajala S, Pärssinen O, Kauppinen M, Koskenvuo M, Kaprio J, Rantanen T. Poor vision accompanied with other sensory impairments as a predictor of falls in older women. Age Ageing 2009; 38(2):162-167.

34. West CG, Gildengorin G, Haegerstrom-Portnoy G, Schneck ME, Lott L, Brabyn J. Is vision function related to physical functional ability in older adults? J Am Geriatr Soc 2002; 50(1):136-145.

35. Deandrea S, Lucenteforte E, Bravi F, Foschi R, La Vecchia C, Negri E. Risk Factors for Falls in Community-dwelling Older People. Epidemiology 2010; 21(5):658-668.

36. Bauer JM, Sieber CC. Sarcopenia and frailty: A clinician's controversial point of view. Exp Gerontol 2008; 43(7):674-678.

37. Chew FLM, Yong CK, Mas Ayu S, Tajunisah I. The association between various visual function tests and low fragility hip fractures among the elderly: A Malaysian experience. Age Ageing 2010; 39(2):239-245.

38. Court H, McLean G, Guthrie B, Mercer SW, Smith DJ. Visual impairment is associated with physical and mental comorbidities in older adults: a cross-sectional study. BMC Med 2014; 12(1):181.

39. Beauchet O, Fantino B, Allali G, Muir SW, Montero-Odasso M, Annweiler C. Timed up and go test and risk of falls in older adults : a systematic review. J Nutr Heal Aging 2011; 15(10):6-11.

40. Schoene D, Wu SM, Mikolaizak AS, Menant JC, Smith ST, Delbaere K, Lord SR. Discriminative ability and predictive validity of the timed up and go test in identifying older people who fall: Systematic review and meta-analysis. J Am Geriatr Soc 2013; 61(2):202-208.

41. Barry E, Galvin R, Keogh C, Horgan F, Fahey T. Is the Timed Up and Go test a useful predictor of risk of falls in community dwelling older adults: a systematic review and meta- analysis. BMC Geriatrics 2014; 14(1):14.

42. Pau M, Leban B, Collu G, Migliaccio GM. Effect of light and vigorous physical activity on balance and gait of older adults. Arch Gerontol Geriatr 2014; 59(3):568573.

43. Hernandez D, Rose DJ. Predicting Which Older Adults Will or Will Not Fall Using the Fullerton Advanced Balance Scale. Arch Phys Med Rehabil 2008; 89(12):23092315.

44. Muir SW, Berg K, Chesworth B, Klar N, Speechley M. Balance impairment as a risk factor for falls in community-dwelling older adults who are high functioning: a prospective study. Phys Ther 2010; 90(3):338-347.

45. Instituto Brasileiro de Geografia e Estatística (IBGE). Pesquisa Nacional de Saúde - 2013: percepção do estado de saude, estilos de vida e doenças crônicas - Brasil Grandes Regiões e Unidades da Federação. Rio de Janeiro: IBGE; 2014

46. Thibaud $\mathrm{M}$, Bloch $\mathrm{F}$, Tournoux-Facon $\mathrm{C}$, Brèque $\mathrm{C}$, Rigaud AS, Dugué B, Kemoun G. Impact of physical activity and sedentary behaviour on fall risks in older people: A systematic review and meta-analysis of observational studies. Eur Rev Aging Phys Act 2012; 9(1):5-15.
47. Oliveira AS, Trevizan PF, Bestetti MLT, Melo RC. Fatores ambientais e risco de quedas em idosos: revisão sistemática. Rev Bras Geriatr e Gerontol 2014; 17(3):637-645.

48. Okubo Y, Seino S, Yabushita N, Osuka Y, Jung S, Nemoto $\mathrm{M}$, et al. Longitudinal association between habitual walking and fall occurrences among community-dwelling older adults: Analyzing the different risks of falling. Arch Gerontol Geriatr 2015; 60(1):45-51.

49. Chang VC, Do MT. Risk Factors for Falls Among Seniors: Implications of Gender. Am J Epidemiol 2015; 181(7):521-531.

50. Foldvari M, Clark M, Laviolette LC, Bernstein MA, Kaliton D, Castaneda C, Pu CT, Hausdorff JM, Fielding RA, Singh MA. Association of Muscle Power With Functional Status in Community-Dwelling Elderly Women. J Gerontol A Biol Sci Med Sci 2000; 55(4):M192-M199.

51. Destro JR, Boing AF, D'Orsi E. Factors associated to medical consultations by elderly adults in southern Brazil: a population based study. Rev Bras Epidemiol 2014; 17(3):692-704.

52. Olofsson P, Lundstrom M, Stenevi U. Gender and referral to cataract surgery in Sweden. Acta Ophthalmolscand 2001; 79(1395-3907):350-353.

53. Tobacman JK, Zimmerman B, Lee P, Hilborne L, Kolder H, Brook RH. Visual function impairments in relation to gender, age, and visual acuity in patients who undergo cataract surgery. Ophthalmology 1998; 105(9):1745-1750.

54. Pohl P, Ahlgren C, Nordin E, Lundquist A, Lundin-Olsson L. Gender perspective on fear of falling using the classification of functioning as the model. Disabil Rehabil 2015; 37(3):214-222.

55. Lee HKM, Scudds RJ. Comparison of balance in older people with and without visual impairment. Age Ageing 2003; 32(6):643-649.

56. Morie M, Reid KF, Miciek R, Lajevardi N, Choong K, Krasnoff JB, Storer TW, Fielding RA, Bhasin S, Lebrasseur NK. Habitual physical activity levels are associated with performance in measures of physical function and mobility in older men. J Am Geriatr Soc 2010; 58(9):1727-1733.

57. Chang SF, Yang R Sen, Lin TC, Chiu SC, Chen ML, Lee HC. The Discrimination of using the short physical performance battery to screen frailty for Community-Dwelling elderly people. J Nurs Scholarsh 2014; 46(3):207-215.

58. Ferriolli E, Perracini MR. Factors associated with falls and recurrent falls in elderly: a population-based study. Rev Bras Geriatr e Gerontol 2007; 1(2):49-60.

59. Gillespie LD, Robertson MC, Gillespie WJ, Sherrington C, Gates S, Clemson LM, Lamb SE. Interventions for preventing falls in older people living in the community. Cochrane database Syst Rev 2012; 9(9):CD007146.

60. Meuleners LB, Fraser ML, Ng J, Morlet N. The impact of first-and second-eye cataract surgery on injurious falls that require hospitalisation: A whole-population study. Age Ageing 2014; 43(3):341-346.

61. Close JCT, Lord SR. Fall assessment in older people. BMJ 2011; 343:d5153. 
62. Tiedemann A, Lord SR, Sherrington C. The development and validation of a brief performance-based fall risk assessment tool for use in primary care. J Gerontol A Biol Sci Med Sci 2010; 65(8):896-903.

63. Araújo Filho A, Salomão SR, Berezovsky A, Cinoto RW, Morales PHA, Santos FR, Belfort Júnior R. Prevalence of visual impairment, blindness, ocular disorders and cataract surgery outcomes in low-income elderly from a metropolitan region of São Paulo--Brazil. Arq Bras Oftalmol 2008; 71(2):246-253.

64. Brasil. Ministério da Saúde (BR). Envelhecimento e saúde da pessoa idosa. Brasília: MS; 2007.

Artigo apresentado em 18/12/2015

Aprovado em 30/08/2016

Versão final apresentada em 02/09/2016 